\title{
Image synthesis for inverse obstacle scattering using the eigenfunction expansion theorem
}

\author{
D. Russell Luke, Burnaby
}

January 4, 2005

\begin{abstract}
In recent years several new inverse scattering techniques have been developed that determine the boundary of an unknown obstacle by reconstructing the surrounding scattered field. In the case of sound soft obstacles, the boundary is usually found as the minimum contour of the total field. In this note we derive a different approach for imaging the boundary from the reconstructed fields based on a generalization of the eigenfunction expansion theorem. The aim of this alternative approach is the construction of higher contrast images than is currently obtained with the minimum contour approach.
\end{abstract}

AMS Subject Classification: 35R30, 35P25, 68U10, 94A08

Key words: inverse problems, scattering theory, image processing, eigenfunction expansion

\section{Introduction}

In the past decade a number of new algorithms have appeared for inverse scattering applications in the resonance region. The strategy common to many modern techniques is that of splitting the original ill-posed nonlinear inverse problem into an ill-posed linear inverse problem, and a well-posed nonlinear problem. Surveys and reviews of these techniques can be found in $[2,10]$. For our purposes, we are primarily interested in techniques that actually reconstruct the field surrounding the unknown scatterer. The two principle algorithms that accomplish this are the method of Kirsch and Kress [6], and the point source method proposed by Potthast [11, 12]. Once the field has been reconstructed at different frequencies and incident field directions, one should in principle have all the information necessary to determine the shape of the scatterer. This begs the question, however, of what one should image. A natural quantity to image is the magnitude of the reconstructed total field. In the case of sound soft scatterers, the total field is zero at the boundary of the obstacle for all frequencies and incident fields, thus the boundary of the obstacle is determined as the minimum contour of the superposition of all total fields. This is the conventional approach, which we call the minimum contour method. In the present work, we propose an alternative to imaging minimum contours which, given enough data, produces higher contrast images than the 
conventional approach. Our method is based on a generalization of the eigenfunction expansion theorem due to Rose and Cheney [16]. Rose and Cheney were interested primarily in scattering in inhomogeneous media, and determining the related scattering potential. We adapt their techniques to the setting of obstacle scattering.

We begin with a brief review of the scattering problem in Section 2. The Generalized Eigenfunction Expansion Theorem for Dirichlet obstacles in two or three dimensions is presented in Section 3. These results are then used in Section 4 to synthesize images of a scatterer, which we compare to conventional minimum contour images.

\section{Setting and Problem Statement}

This discussion concerns scattering of small amplitude, monochromatic, waves from an impenetrable obstacle embedded in an isotropic homogeneous medium. The obstacle is identified by its support $D \subset \mathbb{R}^{m},(m=2$, or 3$)$ which has a connected, piecewise $C^{2}$ boundary $\partial D$. The governing equation for this setting is the Helmholtz equation:

$$
\left[\triangle+\kappa^{2}\right] v(x)=0, \quad x \in \Omega \subset \mathbb{R}^{m},
$$

where $\triangle$ denotes the Laplacian, $\kappa \geq 0$ is the wavenumber, and $\Omega$ is an arbitrary simply connected open set. Solutions to Eq.(1) are complex-valued scalar waves parameterized by $\kappa, v: \Omega \times \mathbb{R}_{+} \rightarrow \mathbb{C}$. The surface of the obstacle, $\partial D\left(D=\mathbb{R}^{m} \backslash \Omega\right)$, can satisfy Dirichlet (sound soft), Neumann (sound hard), or Robin boundary conditions (some mixture of these). Since the theory cited in this work can be applied equally to each of these types of obstacles, this discussion focuses on sound soft obstacles. Specifically, the field $v$ satisfies

$$
\left.v\right|_{\partial D}=0, \quad \text { on } \partial D \text {. }
$$

Denote the incident field satisfying Eq.(1) on $\Omega=\mathbb{R}^{m}$ by $v^{i}: \mathbb{R}^{m} \times \mathbb{R}_{+} \rightarrow \mathbb{C}$. The scattering problem is to find the field $v: \overline{D^{c}} \times \mathbb{R}_{+} \rightarrow \mathbb{C}$ that satisfies Eq.(1)-(2) and

$$
v=v^{i}+v^{s},
$$

where $v^{s}: \overline{D^{c}} \times \mathbb{R}_{+} \rightarrow \mathbb{C}$ satisfies Eq.(1) on $D^{c}$ and the Sommerfeld Radiation Condition:

$$
r^{\frac{m-1}{2}}\left(\frac{\partial}{\partial r}-i \kappa\right) v^{s}(x ; \kappa) \rightarrow 0, \quad r=|x| \rightarrow \infty,
$$

uniformly in all directions.

We call the solution $v$ to the scattering problem the total field. The field $v^{s}$ is a radiating solution to the Helmholtz equation Eq.(1) and is called the scattered field. The scattered field $v^{s}$ satisfies the boundary conditions $v^{s}=-v^{i}$ on $\partial D$. Any such solution to Eq.(1), with Dirichlet boundary conditions on $\partial D$ and radiation conditions Eq.(4) is a solution to the more general exterior Dirichlet problem. Existence and uniqueness of the exterior Dirichlet problem, and hence that of the scattering problem, is a classical result (see [3] and references therein). 
Let $D \subset \mathbb{R}^{m}$ be a closed set with connected $C^{2}$ boundary, and let $v^{s}(\cdot ; \kappa) \in C^{2}\left(D^{c}\right) \cap C\left(\overline{D^{c}}\right)$ satisfy Eq.(1) and Eq.(4). Then Green's formula states that

$$
v^{s}(x ; \kappa)=\int_{\partial D}\left\{v^{s}(y ; \kappa) \frac{\partial \Phi(y, x ; \kappa)}{\partial \nu(y)}-\frac{\partial v^{s}(y ; \kappa)}{\partial \nu(y)} \Phi(y, x ; \kappa)\right\} d s(y), \quad x \in D^{c},
$$

where the normal derivative on $\partial D$ is the limit

$$
\frac{\partial v^{s}(y ; \kappa)}{\partial \nu(y)}:=\lim _{t \rightarrow 0^{+}} \nu(y) \cdot \nabla v^{s}(y-t \nu(y) ; \kappa)
$$

in the direction $\nu(y)$, the unit outward normal to the boundary at $y$. The function $\Phi: \mathbb{R}^{m} \times \mathbb{R}^{m} \times$ $\mathbb{R}_{+} \rightarrow \mathbb{C}$ is the free-space fundamental solution to Eq.(1):

$$
\Phi(x, z ; \kappa):=\left\{\begin{array}{ll}
\frac{i}{4} H_{0}^{(1)}(\kappa|x-z|), & x \neq z, \text { and } m=2 \\
\frac{1}{4 \pi} \frac{e^{i k|x-z|}}{|x-z|}, & x \neq z, \text { and } m=3
\end{array} .\right.
$$

Here $H_{0}^{(1)}$ denotes the zero-th order Hankel function of the first kind. Let $v$ be a solution to the scattering problem at a fixed wavenumber $\kappa$ for a sound soft scatterer with incident wave $v^{i}$ that is an entire solution to Eq.(1), then $v=v^{i}+v^{s}$ where

$$
v^{s}(x ; \kappa)=-\int_{\partial D} \frac{\partial v(y ; \kappa)}{\partial \nu(y)} \Phi(x, y ; \kappa) d s(y), \quad x \in D^{c} .
$$

At large distances from the scatterer, the scattered field $v^{s}$ is described by the asymptotic behavior

$$
v^{s}(x ; \kappa)=\frac{e^{i \kappa|x|}}{|x|^{\frac{(m-1)}{2}}}\left\{v^{\infty}(\hat{x} ; \kappa)+O\left(\frac{1}{|x|}\right)\right\},|x| \rightarrow \infty,
$$

where $\mathbb{S}$ is the unit sphere in $\mathbb{R}^{m}$ :

$$
\mathbb{S}:=\left\{x \in \mathbb{R}^{m}|| x \mid=1\right\} \quad \text { and } \quad \hat{x}:=\frac{x}{|x|} .
$$

The function $v^{\infty}: \mathbb{S} \times \mathbb{R}_{+} \rightarrow \mathbb{C}$ denotes the scattering amplitude, or the far field pattern. The far field pattern has the following explicit representation as a mapping of the total field: [3, Thm. $3.12]$

$$
v^{\infty}(\hat{x} ; \kappa)=-\beta(\kappa) \int_{\partial D} \frac{\partial v(y ; \kappa)}{\partial \nu(y)} e^{-i \kappa \hat{x} \cdot y} d s(y), \quad \hat{x} \in \mathbb{S} .
$$

The scaling factor $\beta$ is a function of the frequency $\kappa$ depending on the setting $\mathbb{R}^{m}$ and is given by [3, Eq.(2.13) and Eq.(3.64)]

$$
\beta(\kappa)=\left\{\begin{array}{ll}
\frac{e^{i \frac{\pi}{4}}}{\sqrt{8 \pi \kappa}}, & m=2 \\
\frac{1}{4 \pi} & m=3
\end{array}, \quad \kappa>0\right.
$$

Our discussion relies on two fundamental incident waves: plane waves and point sources. Plane waves are given by

$$
u^{i}(x, \hat{y}, \kappa):=e^{i \kappa x \cdot \hat{y}}, \quad x \in \mathbb{R}^{m}, \hat{y} \in \mathbb{S}, \kappa \in \mathbb{R}_{+} .
$$


Here $\hat{y} \in \mathbb{S}$, denotes the direction of incidence. The corresponding total and scattered fields are denoted by $u(\cdot, \hat{y}, \kappa): \mathbb{R}^{m} \rightarrow \mathbb{C}$ and $u^{s}(\cdot, \hat{y}, \kappa): \mathbb{R}^{m} \rightarrow \mathbb{C}$ respectively. The second of the fundamental incident fields, point sources, are denoted

$$
w^{i}(x, z ; \kappa):=\Phi(x, z ; \kappa), \quad x, z \in \mathbb{R}^{m}, \kappa \in \mathbb{R}_{+}, x \neq z .
$$

The corresponding total and scattered fields are denoted by $w(\cdot, z, \kappa): \mathbb{R}^{m} \backslash\{z\} \rightarrow \mathbb{C}$ and $w^{s}(\cdot, z, \kappa): \mathbb{R}^{m} \backslash\{z\} \rightarrow \mathbb{C}$ respectively. The total field is the resolvent kernel for the Dirichlet Laplacian on $D^{c}$, that is it satisfies the boundary value problem

$$
\begin{aligned}
& {\left[\triangle+\kappa^{2}\right] w(x, z ; \kappa)=-\delta(x-z), \quad x, z \in D^{c}, m=2 \text { or } 3 ;} \\
& w(x, z ; \kappa)=0, \quad x \in \partial D .
\end{aligned}
$$

Problem Eq.(13) is uniquely solvable [3] with $w=w^{i}+w^{s}$ for the radiating field $w^{s}$ given by

$$
w^{s}(x, z ; \kappa)=-\int_{\partial D} \frac{\partial w(y, z ; \kappa)}{\partial \nu(y)} \Phi(x, y ; \kappa) d s(y), \quad x, z \in D^{c},
$$

(see Eq.(7)). The scattered field $w^{s}$ satisfies Eq.(4). The incident field $w^{i}$ is spatially symmetric,

$$
w^{i}(x, z, \kappa)=w^{i}(z, x, \kappa),
$$

thus $w^{s}$ and $w$ also have this property.

The fields due to scattering from incident plane waves, or incident point sources, form a complete basis of functions. This is the content of the Eigenfunction Expansion Theorem discussed in the next section. We use the duality between point sources and plane waves to derive an inversion operator for determining $D$ from measured far field patterns.

\section{Duality and Generalized Eigenfunction Expansions}

The plane waves and point sources introduced above are dual to each other in the sense that the scattered field at a point $z \in D^{c}$ due to an incident plane wave with direction $-\hat{x}, u^{s}(z,-\hat{x}, \kappa)$, is proportional to the far field at the point $\hat{x}$ due to an incident point source located at $z, w^{\infty}(\hat{x}, z, \kappa)$. The next lemma formally characterizes this relationship.

Lemma 3.1 [Duality and Mixed Reciprocity] Let $w$ satisfy Eq.(13) in $D^{c}(m=2,3)$ and $w=w^{i}+$ $w^{s}$ with the incident point source $w^{i}(x, z ; \kappa)=\Phi(x, z ; \kappa)$, where $\Phi$ is given by Eq.(6). Let $u(z,-\hat{x} ; \kappa)$ solve the scattering problem (Eq.(1)-(4)) with the incident plane wave $u^{i}(z,-\hat{x} ; \kappa)=e^{-i \kappa \hat{x} \cdot z}$, where $\hat{x}=\frac{x}{|x|}$. Then, for $\beta(\kappa)$ given by Eq.(10), the following relation holds as $|x| \rightarrow \infty$ :

$$
w(x, z ; \kappa)=\frac{e^{i \kappa|x|}}{|x|^{(m-1) / 2}}\left\{\beta(\kappa) u(z,-\hat{x} ; \kappa)+O\left(|x|^{-1}\right)\right\} .
$$

Moreover, the fields satisfy the mixed reciprocity relation:

$$
w^{\infty}(\hat{x}, z ; \kappa)=\beta(\kappa) u^{s}(z,-\hat{x} ; \kappa), \quad \hat{x} \in \mathbb{S}, z \in D^{c} .
$$


Proof. By [1, 9.2.3] and the binomial expansion of $|x-z|$ for $|x| \gg|z|$, the fundamental solution for $m=2$ or 3 has the asymptotic behavior

$$
\Phi(x, z ; \kappa)=\frac{e^{i \kappa|x|}}{|x|^{(m-1) / 2}}\left\{\beta(\kappa) e^{-i \kappa z \cdot \hat{x}}+O\left(|x|^{-1}\right)\right\}, \quad|x| \rightarrow \infty, \hat{x}=\frac{x}{|x|},
$$

where $\beta(\kappa)$ is given by Eq.(10). This together with Eq.(14) yields

$$
\begin{aligned}
& w(x, z ; \kappa)= \\
& \frac{e^{i \kappa|x|}}{|x|^{(m-1) / 2}}\left\{\beta(k) e^{-i \kappa \hat{x} \cdot z}-\int_{\partial D} \beta(k) e^{-i \kappa \hat{x} \cdot y} \frac{\partial w(y, z ; \kappa)}{\partial \nu(y)} d s(y)+O\left(|x|^{-1}\right)\right\},
\end{aligned}
$$

as $|x| \rightarrow \infty$ for $x, z \in D^{c}$. Since $u=u^{i}+u^{s}$ solves the scattering problem, Green's formula for the scattered field $u^{s}$ yields

$$
\begin{aligned}
u^{s}(z,-\hat{x}, \kappa) & =-\int_{\partial D}\left(w \frac{\partial u^{s}}{\partial \nu}-u^{s} \frac{\partial w}{\partial \nu}\right) d s+\lim _{R \rightarrow \infty} \int_{R \mathbb{S}}\left(w \frac{\partial u^{s}}{\partial \nu_{\mathbb{S}}}-u^{s} \frac{\partial w}{\partial \nu_{\mathbb{S}}}\right) d s \\
& =\int_{\partial D} u^{s} \frac{\partial w}{\partial \nu} d s \\
& =-\int_{\partial D} u^{i}(y,-\hat{x} ; \kappa) \frac{\partial w(y, z ; \kappa)}{\partial \nu(y)} d s(y),
\end{aligned}
$$

where $R \mathbb{S}$ is the sphere of radius $R, \nu_{\mathbb{S}}$ is the unit outward normal to this sphere, and $u^{i}(y,-\hat{x} ; \kappa)=$ $e^{-i \kappa \hat{x} \cdot y}$. Together Eq.(18) and Eq.(19) yield Eq.(16).

The second statement of the lemma follows from the asymptotic behavior of $w$. From Eq.(8) this is

$$
w(x, z ; \kappa)=\frac{e^{i \kappa|x|}}{|x|^{(m-1) / 2}}\left\{\beta(\kappa) e^{-i \kappa \hat{x} \cdot z}+w^{\infty}(\hat{x}, z ; \kappa)+O\left(\frac{1}{|x|}\right)\right\},
$$

as $|x| \rightarrow \infty, \hat{x}=\frac{x}{|x|}$. Comparing this expansion with Eq.(16) yields Eq.(17).

Remark 3.2 For the case $m=3$, Lemma 3.1 can also be found in [14, Lemma 1.3.1]. The mixed reciprocity relation is a standard identity. See, for example, [7] and [13, Theorems 2.1.4 and 2.2.4] for more detail.

REMARK 3.3 Eq.(19) is the dual to Huygens's principle Eq.(7). In particular,

$$
\int_{\partial D} e^{-i \kappa y \cdot \hat{x}} \frac{\partial w(y, x ; \kappa)}{\partial \nu(y)} d s(y)=\int_{\partial D} \frac{\partial u(y,-\hat{x} ; \kappa)}{\partial \nu(y)} \Phi(x, y ; \kappa) d s(y),
$$

for $x, y \in D^{c}, x \neq y$.

Proposition 3.4 The resolvent kernel Eq.(13) of the Dirichlet Laplacian on $D^{c}$ satisfies

$$
\operatorname{Im} w(x, z, \kappa)=-\kappa|\beta(\kappa)|^{2} \int_{\mathbb{S}} u(x, \hat{y}, \kappa) \overline{u(z, \hat{y}, \kappa)} d s(\hat{y}), \quad \hat{y} \in \mathbb{S} .
$$


Proof. For $R>0$ large enough, Eq.(13) and Green's formula gives

$$
\begin{aligned}
\operatorname{Im} w(x, z, \kappa) & =\operatorname{Im} \int_{\mathbb{B}(R, x) \backslash D} w(y, z, \kappa) \delta(y-x) d y \\
& =-\operatorname{Im} \int_{\mathbb{B}(R, x) \backslash D} w(y, z, \kappa)\left(\triangle+\kappa^{2}\right) \bar{w}(y, x, \kappa) d y \\
& =\operatorname{Im} \int_{\partial(\mathbb{B}(R, x) \backslash D)} \bar{w}(y, x, \kappa) \frac{\partial}{\partial \nu} w(y, z, \kappa)-w(y, z, \kappa) \frac{\partial}{\partial \nu} \bar{w}(y, x, \kappa) d s(y) \\
& =\operatorname{Im} \int_{\partial(\mathbb{B}(R, x) \backslash D)}-\operatorname{Im} \int_{\mathbb{B}(R, x) \backslash D} \bar{w}(y, x, \kappa)\left(\triangle+\kappa^{2}\right) w(y, z, \kappa) d y \\
\quad+\operatorname{Im}(x, z, \kappa) &
\end{aligned}
$$

where $\mathbb{B}(R, x)$ is the ball of radius $R$ centered at $x$ and $\nu$ is the unit outward normal. Since $w=0$ on $\partial D$, this yields

$$
\operatorname{Im} w(x, z, \kappa)=\frac{1}{2} \operatorname{Im} \int_{R \mathbb{S}} \bar{w}(y, x, \kappa) \frac{\partial}{\partial \nu} w(y, z, \kappa)-w(y, z, \kappa) \frac{\partial}{\partial \nu} \bar{w}(y, x, \kappa) d s(y) .
$$

Inserting Eq.(16) for $w$ above gives

$$
\begin{aligned}
\operatorname{Im} w(x, z, \kappa)=\frac{\kappa|\beta(\kappa)|^{2}}{2} \lim _{R \rightarrow \infty} \int_{\mathbb{S}} \frac{1}{R^{m-1}} & \{\bar{u}(x,-\hat{y}, \kappa) u(z,-\hat{y}, \kappa) \\
& \left.+u(x,-\hat{y}, \kappa) \bar{u}(z,-\hat{y}, \kappa)+O\left(R^{-1}\right)\right\} R^{m-1} d s(\hat{y})
\end{aligned}
$$

Now, $\int_{\mathbb{S}} \bar{u}(x,-\hat{y}, \kappa) u(z,-\hat{y}, \kappa) d s(\hat{y}) \in \mathbb{R}$, so in the limit $R \rightarrow \infty$ we have

$$
\operatorname{Im} w(x, z, \kappa)=\kappa|\beta(\kappa)|^{2} \int_{\mathbb{S}} \bar{u}(x,-\hat{y}, \kappa) u(z,-\hat{y}, \kappa) d s(\hat{y}) .
$$

Finally, a change of variables from $-\hat{y}$ to $\hat{y}$ yields Eq.(20).

REMARK 3.5 Proposition 3.1 of [16] establishes a corresponding relation to Eq.(20) for the setting of scattering from inhomogeneous media in $\mathbb{R}^{3}$.

Corollary 3.6 (The Eigenfunction Expansion Theorem) Let $u(x, \hat{y}, \kappa)$ solve the scattering problem, Eq.(1)-(4), for the incident plane wave $u^{i}(z, \hat{y}, \kappa)=e^{i \kappa \hat{y} \cdot z}$ with direction $\hat{y} \in \mathbb{S}$. Then, for $x, z \in D^{c}$,

$$
\delta(x-z)=\frac{-1}{(2 \pi)^{m}} \int_{0}^{\infty} \int_{\mathbb{S}} u(x, \hat{y}, \kappa) \overline{u(z, \hat{y}, \kappa)} d s(\hat{y}) \kappa^{m-1} d \kappa
$$


Proof. Since $w$ satisfying Eq.(13) is self-adjoint, by the spectral theorem for self-adjoint operators (see for example [17, Theorem XI.6.1] and [14, Theorem 1.3.1]), we can write

$$
\frac{1}{\pi} \int_{0}^{\infty} \lim _{\epsilon \rightarrow 0} \operatorname{Im} w(x, z, \sqrt{\lambda+i \epsilon}) d \lambda=\frac{2}{\pi} \int_{0}^{\infty} \operatorname{Im} w(x, z, \kappa) \kappa d \kappa=\delta(x-z),
$$

where $\lambda=\kappa^{2}$. Also, by Eq.(10) we have $\frac{\kappa}{\pi}|\beta(\kappa)|^{2}=\frac{\kappa^{m-2}}{2(2 \pi)^{m}}$ for $\kappa>0, m=2$ or 3, thus Eq.(22) and Eq.(20) yield Eq.(21). For more details and a review see [8, Example 4.49], [14, Theorem 1.3.1], or [15].

The next theorem is a generalization of Eq.(21) and relies on additional physical assumptions, namely conjugate symmetry with respect to the frequency $\kappa$ and causality. Before stating the theorem, these assumptions are briefly discussed.

The focus of this discussion has been on implicitly time-harmonic, monochromatic (single frequency, $\kappa>0$ ) waves, that is, the waves take the form $V(x, t)=v(x ; \kappa) e^{i \kappa t}$. In this case only the real part of $V$ is physically relevant. If we drop the assumption that the fields are time-harmonic, then the wave $v$ satisfying Eq.(1) is the Fourier transform of the real-valued function $V: \mathbb{R}^{m} \times \mathbb{R} \rightarrow \mathbb{R}$, thus it is a Hermitian function of the frequency $\kappa \in \mathbb{R}$ :

$$
v(x, k)=\bar{v}(x,-k) .
$$

To accommodate negative frequencies in the time-harmonic case, we require that the function $v$ be a Hermitian function with respect to $\kappa$. With this extension, the integral with respect to $\kappa$ in Eq.(21) can be written

$$
\begin{aligned}
\delta(x-z) & =\frac{-1}{2(2 \pi)^{m}} \int_{\mathbb{R}} \int_{\mathbb{S}} u(x, \hat{y}, \kappa) \overline{u(z, \hat{y}, \kappa)} d s(\hat{y}) \kappa^{m-1} d \kappa \\
& =\frac{-1}{(2 \pi)^{m}} \int_{\mathbb{R}^{m}} u(x, \xi) \overline{u(z, \xi)} d \xi,
\end{aligned}
$$

where $\xi=(\hat{y}, \kappa)$ and $d \xi=\kappa^{m-1} d \kappa d s(\hat{y})$. Here we have used the fact that $u(x, \hat{y}, \kappa) \overline{u(z, \hat{y}, \kappa)}=$ $u(x, \hat{y},-\kappa) \overline{u(z, \hat{y},-\kappa)}$, which follows from the symmetry of the delta function and the conjugate symmetry of $u$. The scaling $\beta$ defined in Eq.(10) must also be modified for negative frequencies. In three dimensions nothing changes, but in two dimensions it is defined as

$$
\beta(\kappa)=\frac{e^{i \frac{\pi}{4}}}{\sqrt{8 \pi|\kappa|}}, \quad m=2, \kappa \neq 0 .
$$

The second physical assumption we must introduce, causality, is formulated as follows:

$$
W(x, z, t)=0 \quad \text { for } t<|x-z|,
$$

where, using the distributional definition of the Fourier transform, $W$ is given by

$$
W(x, z, t)=\frac{1}{\sqrt{2 \pi}} \int_{\mathbb{R}} e^{i \kappa t} w(x, z, k) d \kappa,
$$


and satisfies the Fourier dual of Eq.(13), namely

$$
\begin{gathered}
{\left[\triangle+\frac{\partial^{2}}{\partial t^{2}}\right] W(x, z ; t)=-\delta(x-z) \delta(t), \quad x, z \in D^{c}, m=2 \text { or } 3 ;} \\
W(x, z ; t)=0, \quad x \in \partial D .
\end{gathered}
$$

The statement and proof of the following theorem are modeled after [16, Theorem 3.2].

Theorem 3.7 (Generalized Eigenfunction EXPANsion) Let $w$ satisfy

Eq.(13) in $D^{c}(m=2,3)$ and $w=w^{i}+w^{s}$ with the incident point source $w^{i}(x, z ; \kappa)=\Phi(x, z ; \kappa)$, where $\Phi$ is given by Eq.(6). Let $u(z, \hat{y} ; \kappa)$ solve the Scattering Problem (Eq.(1)-(4)) for the incident plane wave $u^{i}(z, \hat{y} ; \kappa)=e^{i \kappa \hat{y} \cdot z}$ with direction $\hat{y} \in \mathbb{S}$. Define $w$ and $u$ for $\kappa<0$ by the Hermitian extensions

$$
w(x, z, \kappa)=\overline{w(x, z,-\kappa)}, \quad u(x, \hat{y}, \kappa)=\overline{u(x, \hat{y},-\kappa)},
$$

and assume further that the fields are causal as defined by Eq.(25). Then, for any $\tau$ satisfying $|\tau| \leq|x-z| \quad\left(x, z \in D^{c}, \quad x \neq z\right)$

$$
w(x, z, 0)=-\frac{(2 \pi)^{-m}}{2} \int_{\mathbb{R}} \kappa^{m-3} e^{i \kappa \tau} \int_{\mathbb{S}} u(x, \hat{y}, \kappa) \overline{u(z, \hat{y}, \kappa)} d s(\hat{y}) d \kappa,
$$

where the integral is in the sense of distributions with respect to $z$ :

$$
\begin{aligned}
& \int_{D^{c}} w(x, z, 0) \varphi(z) d z= \\
& \quad-\frac{(2 \pi)^{-m}}{2} \int_{\mathbb{R}} \kappa^{m-3} \int_{\mathbb{S}} u(x, \hat{y}, \kappa) \int_{D^{c}} \overline{u(z, \hat{y}, \kappa)} e^{i \kappa \tau} \varphi(z) d z d s(\hat{y}) d \kappa,
\end{aligned}
$$

for $\varphi(z) \in C_{0}^{\infty}\left(\mathbb{R}^{m}\right)$.

Proof. Consider the distribution in $\tau$

$$
q(x, z, \tau)=-\frac{(2 \pi)^{-m}}{2} \int_{\mathbb{R}} \kappa^{m-3} e^{i \kappa \tau} \int_{\mathbb{S}} u(x, \hat{y}, \kappa) \overline{u(z, \hat{y}, \kappa)} d s(\hat{y}) d \kappa
$$

where this integral is interpreted as

$$
\begin{aligned}
\int_{\mathbb{R}} q(x, z, \tau) \varphi(\tau) d \tau & = \\
& -\frac{(2 \pi)^{-m}}{2} \int_{\mathbb{R}} \varphi(\tau) \int_{\mathbb{R}} \kappa^{m-3} e^{i \kappa \tau} \int_{\mathbb{S}} u(x, \hat{y}, \kappa) \overline{u(z, \hat{y}, \kappa)} d s(\hat{y}) d \kappa d \tau
\end{aligned}
$$

for $\varphi(\tau) \in C_{0}^{\infty}(\mathbb{R})$. By Eq.(23) this can be rewritten as

$$
\begin{aligned}
\int_{\mathbb{R}} q & (x, z, \tau) \varphi(\tau) d \tau \\
& =-\frac{(2 \pi)^{-m}}{2} \int_{\mathbb{R}} \varphi(\tau) \int_{\mathbb{R}} \kappa^{m-3} e^{i \kappa \tau} \frac{2(2 \pi)^{m} \kappa^{2-m}}{\pi} \operatorname{Im} w(x, z, \kappa) d \kappa d \tau \\
& =-\frac{1}{\pi} \int_{\mathbb{R}} \varphi(\tau) \int_{\mathbb{R}} \kappa^{-1} e^{i \kappa \tau} \operatorname{Im} w(x, z, \kappa) d \kappa d \tau .
\end{aligned}
$$


We now substitute the inverse of Eq.(26),

$$
w(x, z, \kappa)=\frac{1}{\sqrt{2 \pi}} \int_{\mathbb{R}} e^{-i \kappa t} W(x, z, t) d t,
$$

and use the fact that $W$ is real-valued, together with an interchange in the order of integration, to obtain

$$
\begin{aligned}
& -\frac{1}{\pi} \int_{\mathbb{R}} \varphi(\tau) \int_{\mathbb{R}} \kappa^{-1} e^{i \kappa \tau} \operatorname{Im} w(x, z, \kappa) d \kappa d \tau \\
& \quad=-\frac{1}{\pi} \int_{\mathbb{R}} \varphi(\tau) \int_{\mathbb{R}} \kappa^{-1} e^{i \kappa \tau} \operatorname{Im} \frac{1}{\sqrt{2 \pi}} \int_{\mathbb{R}} W(x, z, t) e^{-i \kappa t} d t d \kappa d \tau \\
& \quad=-\int_{\mathbb{R}} \varphi(\tau) \int_{\mathbb{R}} \kappa^{-1} e^{i \kappa \tau} \frac{1}{2 \pi i \sqrt{2 \pi}} \int_{\mathbb{R}} W(x, z, t)\left(e^{-i \kappa t}-e^{i \kappa t}\right) d t d \kappa d \tau \\
& \quad=-\int_{\mathbb{R}} \varphi(\tau) \frac{1}{\sqrt{2 \pi}} \int_{\mathbb{R}} W(x, z, t) \frac{1}{2 \pi i} \int_{\mathbb{R}}(\kappa)^{-1}\left(e^{i \kappa(\tau-t)}-e^{i \kappa(\tau+t)}\right) d \kappa d t d \tau .
\end{aligned}
$$

The principal part of the integral with respect to $\kappa$ is

$$
\frac{1}{2 \pi i} \int_{\mathbb{R}}(\kappa)^{-1}\left(e^{i \kappa(\tau-t)}-e^{i \kappa(\tau+t)}\right) d \kappa=\operatorname{sgn}(\tau-t)-\operatorname{sgn}(\tau+t) .
$$

Thus

$$
\begin{aligned}
-\frac{1}{\pi} \int_{\mathbb{R}} & \varphi(\tau) \int_{\mathbb{R}} \kappa^{-1} e^{i \kappa \tau} \operatorname{Im} w(x, z, \kappa) d \kappa d \tau \\
= & -\int_{\mathbb{R}} \varphi(\tau) \frac{1}{\sqrt{2 \pi}} \int_{\mathbb{R}} W(x, z, t)(\operatorname{sgn}(\tau-t)-\operatorname{sgn}(\tau+t)) d t d \tau \\
= & \int_{\mathbb{R}} \varphi(\tau) \frac{1}{\sqrt{2 \pi}}\left(-\int_{-\infty}^{-\tau} W(x, z, t) d t+\int_{\tau}^{\infty} W(x, z, t) d t\right) d \tau \\
= & \int_{\mathbb{R}} \varphi(\tau) \frac{1}{\sqrt{2 \pi}} \int_{|x-z|}^{\infty} W(x, z, t) d t d \tau \\
= & \int_{\mathbb{R}} \varphi(\tau) \frac{1}{\sqrt{2 \pi}} \int_{-\infty}^{\infty} W(x, z, t) d t d \tau .
\end{aligned}
$$

In the last two equalities above we used causality and the assumption that $|\tau|<|x-z|$. Now by Eq. (26)

$$
\frac{1}{\sqrt{2 \pi}} \int_{-\infty}^{\infty} W(x, z, t) d t=w(x, z, 0)
$$

thus $q(x, z, \tau)=w(x, z, 0)$ since

$$
\int_{\mathbb{R}} q(x, z, \tau) \varphi(\tau) d \tau=\int_{\mathbb{R}} w(x, z, 0) \varphi(\tau) d \tau
$$

which completes the proof. 
COROLlary 3.8 Under the assumptions of Theorem 3.7, for any $\tau(x, z)$ satisfying $|\tau(x, z)| \leq$ $|x-z| \quad\left(x, z \in D^{c}, \quad x \neq z\right)$ we have

$$
\delta(x-z)=-\frac{(2 \pi)^{1-m}}{2} \int_{\mathbb{R}} \kappa^{m-3} \int_{\mathbb{S}} \triangle\left(e^{i \kappa \tau(x, z)} u(x, \hat{y}, \kappa) \overline{u(z, \hat{y}, \kappa)}\right) d s(\hat{y}) d \kappa,
$$

where the Laplacian $\triangle$ may be with respect to either variable, $x$ or $z$. The integral is understood in the sense of distributions:

$$
\begin{aligned}
\varphi(x) & =\int_{D^{c}} \delta(x-z) \varphi(z) d z= \\
& -\frac{(2 \pi)^{-m}}{2} \int_{\mathbb{R}} \kappa^{m-3} \int_{\mathbb{S}} \triangle u(x, \hat{y}, \kappa) \int_{D^{c}} \overline{u(z, \hat{y}, \kappa)} e^{i \kappa \tau(x, z)} \varphi(z) d z d s(\hat{y}) d \kappa,
\end{aligned}
$$

for $\varphi(z) \in C_{0}^{\infty}\left(\mathbb{R}^{m}\right)$.

Proof. The proof follows from Eq.(13) and Eq.(28). The proof for the corresponding statement for the setting of inhomogeneous media in $\mathbb{R}^{3}$ in [16, Corollary 3.3] carries over to the present setting.

The parameter $\tau(x, z)$ is more general than we need. Let

$$
\tau(x, z)=\hat{\eta} \cdot x-\hat{\eta} \cdot z .
$$

For this choice of $\tau$, it is more suggestive to rewrite Eq.(30) as

$$
\varphi(x)=-\frac{(2 \pi)^{-m}}{2} \triangle\left(\int_{\mathbb{R}} \kappa^{m-3} \int_{\mathbb{S}} u(x, \hat{y}, \kappa) e^{i \kappa \hat{\eta} \cdot x} \tilde{\varphi}(\hat{\eta}, \hat{y}, \kappa) d s(\hat{y}) d \kappa\right),
$$

where

$$
\tilde{\varphi}(\hat{\eta}, \hat{y}, \kappa)=\int_{D^{c}} \overline{u(z, \hat{y}, \kappa)} e^{-i \kappa \hat{\eta} \cdot z} \varphi(z) d z
$$

In the setting of inhomogenious media, the asymptotic expansion of the Lippmann-Schwinger equation for the total field [3, Eq.(8.27)] yields

$$
\int_{\mathbb{R}^{3}} \overline{u(z, \hat{y}, \kappa)} e^{-i \kappa \hat{\eta} \cdot z} \bar{m}(z) d z=-\frac{1}{\beta(\kappa) \kappa^{2}} \overline{u^{\infty}}(-\hat{\eta}, \hat{y}, \kappa)
$$

for $m(z)=1-n(z)$, where $n: \mathbb{R}^{3} \rightarrow \mathbb{C}$ is the index of refraction. Thus, for $\varphi(z)=\bar{m}(z)$ in Eq.(32) with $D^{c}=\mathbb{R}^{3}$, we have

$$
\tilde{\varphi}(\hat{\eta}, \hat{y}, \kappa)=-\frac{1}{\beta(\kappa) \kappa^{2}} \overline{u^{\infty}}(-\hat{\eta}, \hat{y}, \kappa)
$$

In the present setting of scattering from impenetrable obstacles, the connection between the density $\varphi$ and the far field pattern is not clear. Nevertheless, Eq.(31) offers an expression for numerically calculating the density $\varphi$ that satisfies the integral equation

$$
-\frac{1}{\beta(\kappa) \kappa^{2}} \overline{u^{\infty}}(-\hat{\eta}, \hat{y}, \kappa)=\int_{D^{c}} \overline{u(z, \hat{y}, \kappa)} e^{-i \kappa \hat{\eta} \cdot z} \varphi(z) d z .
$$


It is also unclear how Eq.(31) behaves at points on the interior of $D$. Inverse scattering methods that calculate the field surrounding the obstacle also calculate a field inside the scatterer. Such fields are purely numerical artifacts, but, without knowing the shape and location of the obstacle, it is not possible to distinguish numerical artifacts from reconstructed field values. Fortunately, however, the inconsistency between the behavior of the numerical artifacts and that of proper scattered waves shows up quite dramatically in numerical computations. In the next section we compute Eq.(31) for $\tilde{\varphi}$ satisfying Eq.(34) using the field $u$ in Eq.(31) estimated by an application of the point source method introduced by Potthast $[11,12]$.

\section{Numerical Results}

In this section we apply Corollary 3.8 to the synthesis of the shape of a sound soft scatterer, shown in Figure 1, once the total field around the scatterer has been determined. We use the point source method to reconstruct, from far field measurements, the total field along rays centered on the obstacle. Since this implementation of the point source method is novel, we give a brief sketch of the technique below. Once the total field for various frequencies and incident field directions is known, the final image of the scatterer is constructed by computing the integral Eq.(31) with $\tilde{\varphi}$ satisfying Eq.(34). Figures 2(e)-(h) show the absolute value of the potential $\varphi$. This method is compared to the minimum contour approach, shown in Figures 2(a)-(d), which amounts to calculating the integral of the absolute value of the total field over all directions and frequencies:

$$
\varphi(x)=\int_{\mathbb{R}} \int_{\mathbb{S}}|u(x, \hat{y}, \kappa)| d s(\hat{y}) d \kappa .
$$

The minimum contour approach determines the boundary of the scatterer as those points $x$ where $\varphi(x)$ falls below a given tolerance.

As described in $[11,12,13]$ and more recently in [9], the point source method uses a predetermined approximating domain, denoted by $D_{a}$, in place of the true scatterer boundary in order to reconstruct the scattered field around the obstacle. The accuracy of the reconstruction at some point $z \in{\overline{D^{c}}}_{a}$ depends first on whether or not the obstacle lies on the interior of $D_{a}$, and then on how accurately one solves a simple ill-posed inverse problem of approximating the field along $\partial D_{a}$ due to a point source located at the fixed point $z$. The points $z$ where an accurate reconstruction is achieved depends principally on the size and shape of the approximating domain $D_{a}$ as well as the location of $z$ relative to $D_{a}$.

For our reconstructions we assume two a priori pieces of information, namely that the center of the obstacle and it's approximate size are known. In general one could estimate these by further manipulations of the point source method, but for simplicity we take these as given. We chose $D_{a}$ to be a circle twice as large as the obstacle $D$ and fix the point $z$ just outside $D_{a}$ with orientation, or direction, $\hat{\eta}$ from the center. For this geometry of the approximating domain and reference point $z$ it can be shown that the most accurate reconstructions of the scattered field lie along rays with direction $\hat{\eta}$ relative to the center of the obstacle. It is reasonable, then, to reconstruct the field on a polar grid. A more detailed description of this behavior is beyond the scope of this work. Our 


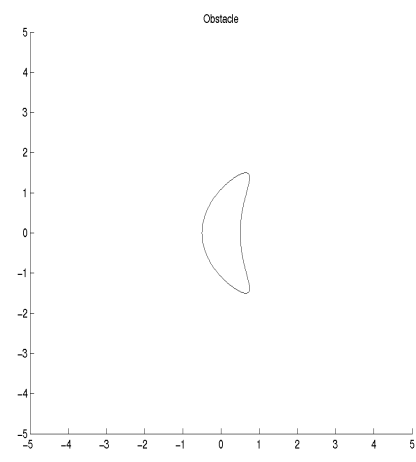

Figure 1: Obstacle used in experiments. The parametric description of this "kite-shaped" object is described in [3].

purpose is merely to motivate why we reconstruct the scattered field on a polar grid along rays extending from the center of the obstacle.

We determine the scattered field at 20 frequencies, equally spaced on the interval $[-8,8]$, and at 32 different incident field directions equally spaced on $[-\pi, \pi]$. Since the field is reconstructed on a polar grid, we must use the polar Laplacian in Eq.(31),

$$
\triangle u(r, \theta)=\frac{\partial^{2} u}{\partial r^{2}}+\frac{1}{r^{2}} \frac{\partial^{2} u}{\partial \theta^{2}}+\frac{1}{r} \frac{\partial u}{\partial r} .
$$

The modulus of the images calculated by the two methods are compared for varying amounts of data, from single frequency and incident field direction, to multiple frequency and incident field directions. In the first instance, the two methods appear qualitatively the same for single frequency and incident field, as would be expected since Figure 2(e) is essentially the Laplacian of Figure 2(a). In the case of multiple frequency and incident field directions the image obtained by Eq.(31) and Eq.(34), Figure 2(h), are sharper and have greater contrast than the minimum contour counterpart Figure 2(d).

\section{Concluding Remarks}

The numerical demonstration above indicates that, where there is plenty of data available, the Generalized Eigenfunction Expansion Theorem can be exploited to achieve high contrast images for determining the shape of obstacles from far field data. In limited data situations it is not clear that this technique is preferable to the conventional minimum contour approach. Nevertheless, we observed that the dynamic range of the images constructed via Eq.(31) and Eq.(34) is several orders of magnitude greater than that for the minimum contour images. This is to be expected from the Laplacian in Eq.(31). Not shown in Figure 2 is a sharp singularity that develops at the center of the image reconstruction. The location and appearance of this singularity depends on our choice of center for the obstacle. For any center that is interior to the actual scatterer, the 
(a)

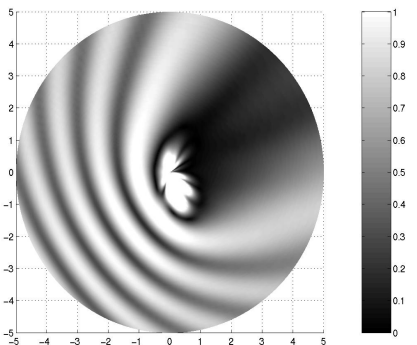

(b)
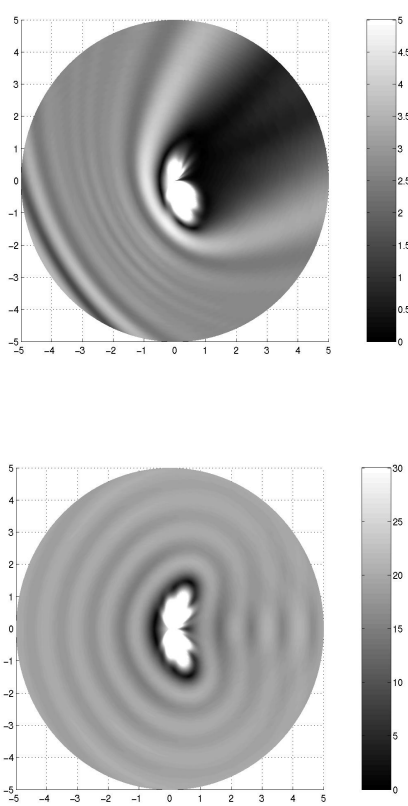

(c)

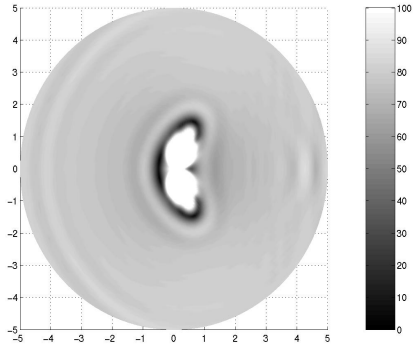

(d) (e)

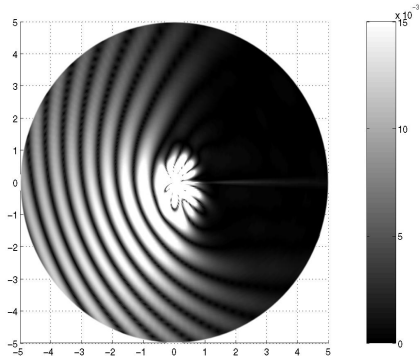

(f)

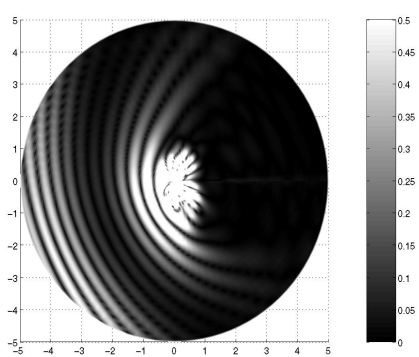

(h)

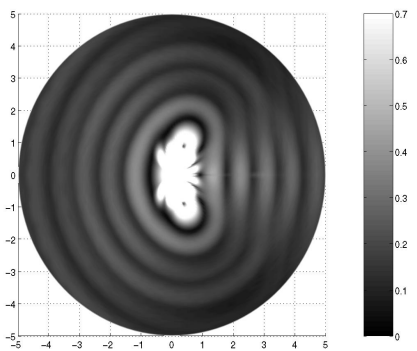

(i)

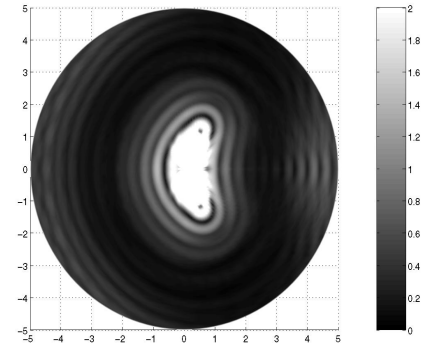

Figure 2: Comparison of image synthesis techniques for limited data. Frames (a)-(d) are images obtained using Eq.(36); (e)-(h) are obtained using Eq.(31) and Eq.(34). The top row, (a) and (e), are images corresponding to a single incident field direction and a single frequency; images (b) and (f) correspond to 20 frequencies and a single incident direction; images (c) and (g) correspond to a single frequency and 32 incident directions; the last row, (d) and (h), correspond to 20 frequencies and 32 incident field directions. 
reconstructed image has a sharp singularity at the chosen central point. If the central point is on the exterior of the scatterer, then there is no singularity at the center point of the reconstructed image. This suggests a type of "response test" for determining the interior of the scatterer as those points where the calculated numerical value is "large". This is analogous, though complementary, to the no response test described in [10].

\section{References}

[1] M. Abramowitz and I. A. Stegun: Handbook of Mathematical Functions, 9th Edition (Dover, New York, 1972).

[2] D. Colton, J. Coyle, and P. Monk: Recent Developments in Inverse Scattering Theory. SIAM Review 42 (2000), 3, 369-414.

[3] D. Colton and R. Kress: Inverse Acoustic and Electromagnetic Scattering Theory, 2nd Ed. (Springer-Verlag, Berlin, 1998).

[4] D. Colton and P. Monk: A novel method for solving the inverse scattering problem for timeharmonic waves in the resonance region. SIAM Journal on Applied Mathematics, 45 (1985), 1039-1053.

[5] D. Colton and P. Monk: A novel method for solving the inverse scattering problem for timeharmonic waves in the resonance region II. SIAM Journal on Applied Mathematics, 46 (1986), 506-523.

[6] A. Kirsch and R. Kress: A numerical method for an inverse scattering problem, in Inverse Problems, Engl and Groetsch, eds. (Academic Press, Orlando, 1987), pg.279-290.

[7] R. Kress: Integral Equation Methods in Inverse Acoustic and Electromagnetic Scattering, in Boundary Integral Formulations for Inverse Analysis, D. B. Ingham and L. C. Wrobel eds. (Computational Mechanics Publications, South Hampton, UK, 1997).

[8] R. Leis: Initial-boundary value problems in mathematical physics (B. G. Teubner, Stuttgart; John Wiley \& Sons, Ltd., Stuttgart, 1986).

[9] D. R. Luke: Multifrequency inverse obstacle scattering: the point source method and generalized filtered backprojection. Mathematics and Computers in Simulation, (in press).

[10] D. R. Luke and R. and Potthast: The no response test - a sampling method for inverse scattering problems, SIAM Journal on Applied Mathematics, 63 (2003), 4, 1292-1312.

[11] R. Potthast: A fast new method to solve inverse scattering problems, Inverse Problems, 12 (1996), 731-742.

[12] R. Potthast: A point-source method for inverse acoustic and electromagnetic obstacle scattering problems, IMA Jour. Appl. Math. 61, 119-140 (1998). 
[13] R. Potthast: Point sources and multipoles in inverse scattering theory (Chapman \& Hall, London, 2001).

[14] A. G. Ramm: Scattering by Obstacles. (D. Reidel Publishing, Dordrecht, Holland, 1986).

[15] M. Reed and B. Simon: Methods of Modern Mathematical Physics III, Scattering Theory. (Academic Press, New York, 1979).

[16] J. H. Rose and M. Cheney: Generalized eigenfunction expansions for scattering in inhomogeneous three-dimensional media. Journal of Mathematical Physics, 29 (1988), 1347-1355.

[17] Yosida, K. Functional Analysis, 2nd ed. (Springer, Berlin, 1968).

D. Russell Luke

Department of Mathematics, Simon Fraser University

Burnaby, British Columbia

Canada

drluke@pims.math.ca

This work was supported by a postdoctoral fellowship from the Pacific Institute for the Mathematical Sciences. 